\title{
Hans-Dieter Anders
}

Kunibert Schwarz

\section{Modelle}

zur Ermittlung

eines optimalen

Verhältnisses

von Akkumulation

und Konsumtion

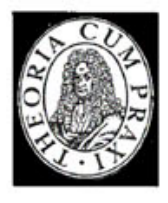

Akademie-Verlag $\cdot$ Berlin 
In den Forschungsberichten des Zentralinstituts fürWirtschaftswissenschaften werden die jeweils neuesten Arbeitsergebnisse zu aktuellen Problemen der politischen Ökonomie des Sozialismus sowie zur Auseinandersetzung mit der heutigen bürgerlichen Ökonomie veröffentlicht. Die Forschungsberichte sollen nicht nur über neue Forschungen informieren; sie sollen vor allem zur Diskussion über die zielgerichtete Fortführung der Arbeiten anregen.

Der Herausgeber 
Modelle zur Ermittlung eines optimalen Verhältnisses von Akkumulation und Konsumtion 
Forschungsberichte

herausgegeben von dem

Zentralinstitut für Wirtschaftswissenschaften der Akademie der Wissenschaften der DDR, Direktor Prof. Dr. habil. Wolfgang Heinrichs Nr. 19 
HANS-DIETER ANDERS

KUNIBERT SCHWARZ

\section{Modelle zur Ermittlung}

eines optimalen Verhältnisses

von Akkumulation

und Konsumtion

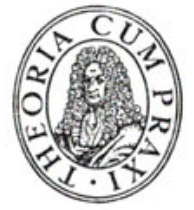

AKADEMIE-VERLAG · BERLIN 1976 


\author{
Autoren: \\ Dr. Hans-Dieter Anders \\ Dr. Kunibert Schwarz \\ unter Mitarbeit von: \\ Dr. Helmut Steckler \\ Dr. Peter Trettin
}

Wissenschaftlicher Redakteur:

Dr. Walburga Wicke

Erschienen im Akademie-Verlag, 108 Berlin, Leipziger Str. 3-4

(C) Akademie-Verlag, Berlin 1976

Lizenznummer: $202 \cdot 100 / 46 / 76$

Gesamtherstellung: VEB Druckerei „Thomas Müntzer“,

582 Bad Langensalza

Bestellnummer: 7531888 (2163/19) · LSV 0345

Printed in GDR

DDR 5,- M 